\title{
When learning about the real world is better done virtually: A study of substituting computer simulations for laboratory equipment
}

\author{
N. D. Finkelstein, W. K. Adams, C. J. Keller, P. B. Kohl, K. K. Perkins, N. S. Podolefsky, and S. Reid \\ Department of Physics, University of Colorado, Boulder, Colorado 80309, USA \\ R. LeMaster \\ Kavli Operating Institute, Santa Barbara, California 93101, USA
}

(Received 17 May 2005; published 6 October 2005)

\begin{abstract}
This paper examines the effects of substituting a computer simulation for real laboratory equipment in the second semester of a large-scale introductory physics course. The direct current circuit laboratory was modified to compare the effects of using computer simulations with the effects of using real light bulbs, meters, and wires. Two groups of students, those who used real equipment and those who used a computer simulation that explicitly modeled electron flow, were compared in terms of their mastery of physics concepts and skills with real equipment. Students who used the simulated equipment outperformed their counterparts both on a conceptual survey of the domain and in the coordinated tasks of assembling a real circuit and describing how it worked.
\end{abstract}

DOI: 10.1103/PhysRevSTPER.1.010103

PACS number(s): 01.40.Fk, 01.50.Ht

\section{INTRODUCTION}

Since the introduction of the microcomputer several decades ago, researchers and educators have developed, explored, and studied mechanisms for employing computers in the classroom. Over this time, computers have made their way into nearly every element of university courses in physics. Microcomputers have been used to augment large-scale lectures, ${ }^{1}$ to promote small-group and individual student work, ${ }^{2}$ and most areas in between. In the laboratory, computers have served as minor additions to collect or display data, as a means of modifying laboratory experience, ${ }^{3}$ or as complete virtual worlds in which students embed themselves. ${ }^{4,5}$

Appropriately, Turkle questions the motives and justification for use of computers in education: ${ }^{6}$

"Why should fifteen-year-olds pour virtual chemicals into virtual beakers? Why should eighteen-year-olds do virtual experiments in virtual physics laboratories? The answer to these questions is often: because the simulations are less expensive; because there are not enough science teachers. But these answers beg a large question: Are we using computer technology not because it teaches best but because we have lost the political will to fund education adequately?"

We begin to tackle Turkle's questions by asking whether and in what fashion computers might be the best educational option for our students. Commonly, computers serve as an integral part of recitations or laboratories, for real or virtual investigations in introductory physics courses..$^{2,3,7-10}$ These applications have included using computers to facilitate data acquisition, to provide real-time data display, to analyze these data, and to simulate complex phenomena. Such efforts have been shown to be as effective as or more effective than their non-computer-based counterparts, be they traditional ${ }^{9,11}$ or physics-education-research-based activities. ${ }^{2,3,7}$ In focusing our attention directly on the impact of the use of com- puter simulations in lieu of real equipment in physics laboratories, we find relatively few studies. Zacharia and Anderson, ${ }^{11}$ in a small study of university students' use of computers to prepare for laboratories, found that students made greater conceptual gains when using the computer to prepare for laboratory than those who used the textbook and solved additional problems on the topic. Linn and colleagues ${ }^{12,13}$ have demonstrated that using the computer as a learning partner (to substitute for laboratory equipment, to collect and display data, and to serve as a medium of communication and coordination of students and teachers) supports students' mastery of concepts and ability to integrate knowledge. In a direct comparison substituting computer simulations and video for hands-on equipment in an elementary school class, Triona and Klahr ${ }^{14}$ demonstrated that computer simulations can be as productive a learning tool as hands-on equipment, given the same curricula and educational setting.

Here, we examine the effectiveness of completely replacing traditional equipment with computer-based simulations. Given the constraints (e.g., large expense) of traditional laboratories and the related concerns of computer limitations (e.g., students miss out on the hands-on experiences with equipment), we explore whether it is possible to achieve the conceptual learning gains and mastery of mechanical skills associated with real equipment by instead working with computer simulations. We extend prior work by examining the following research questions:

(1) Can simulations be used productively in lieu of real equipment in undergraduate labs?

(2) Will students learn the same concepts and will they learn them as well?

(3) What is lost? Will students develop an understanding of using real equipment by working with simulations?

Our results indicate that properly designed simulations used in the right contexts can be more effective educational tools than real laboratory equipment, both in developing stu- 


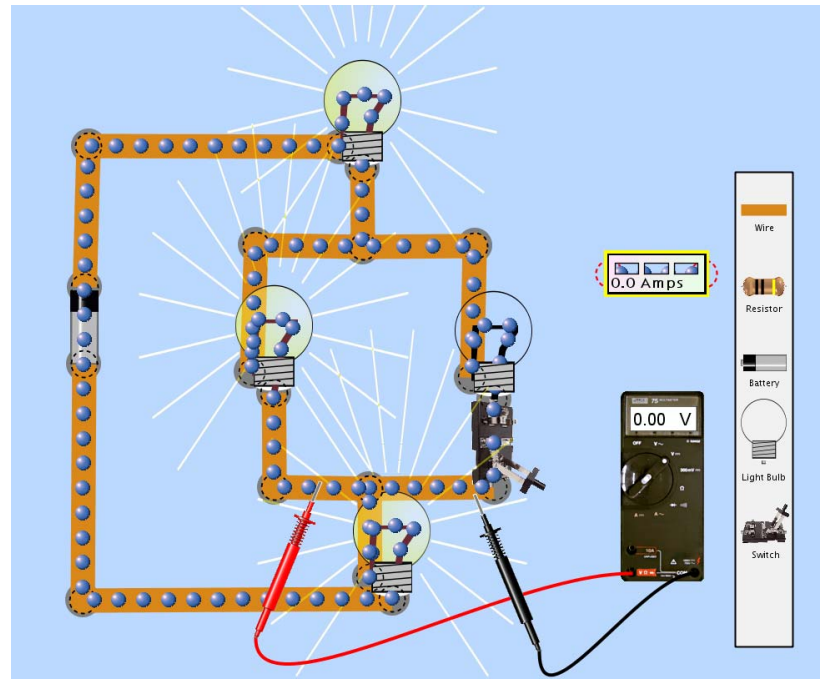

FIG. 1. (Color) Screenshot of the Physics Education Technology project simulation, Circuit Construction Kit.

dent facility with real equipment and at fostering student conceptual understanding.

\section{The physics education technology simulations}

The Physics Education Technology (PhET) project at the University of Colorado has developed a suite of physics simulations that take advantage of the opportunities of computers while addressing some of the limiting concerns of these tools. ${ }^{15}$ The PhET project has developed approximately 50 simulations that span the curriculum of introductory physics and are freely available online. ${ }^{16}$ These simulations are designed to be highly interactive, engaging, and open learning environments that provide animated feedback to the user. The simulations model physically accurate, highly visual, dynamic representations of physics principles. Simultaneously, the simulations are designed to build explicit bridges between students' everyday understanding of the world and its underlying physical principles, often by making these physical models (such as current flow or electric field lines) visible. For instance, a student learning about electromagnetic radiation starts with a radio station transmitter and antenna at a nearby house. Students can drive an electron to oscillate at the transmission station, then observe the propagation of the electric field and the resulting motion of an electron at the receiving antenna. A variety of virtual observation and measurement tools are provided to encourage students to explore properties of this microworld. ${ }^{17}$ Each simulation is tested with multiple student interviews before being user tested in class and out of class. Knowledge gained from these evaluations is iteratively used to improve the next generation of simulations. More on the PhET project and the research methods used to develop the simulations is available in Ref. 16.

The Circuit Construction Kit (CCK) simulates the behavior of simple electric circuits and provides an open workspace where students can manipulate resistors, light bulbs, wires, and batteries (Fig. 1). Each element has operating pa- rameters (such as resistance or voltage) that can be varied by the user and measured by a simulated voltmeter and ammeter. Current and voltage are calculated throughout the circuit using Kirchhoff's laws. The batteries and wires are designed to operate either as ideal components or as real components by including appropriate, finite resistance. The light bulbs are modeled as Ohmic in order to emphasize the basic models of circuits that are presented in introductory physics courses. Moving electrons are explicitly shown to visualize current flow and current conservation. The explicit use of moving electrons as a visual cue of current flow can be considered an attempt to provide a microscopic model; however, we believe this to be an intermediate state in the debates on whether to teach microscopic or macroscopic models of physics $^{18,19}$ and will be the subject of future investigation. ${ }^{20}$ Much attention has been placed on the user interface (UI) to ensure that users can interact easily with the simulation. The $\mathrm{UI}$ is also designed to encourage users to engage with concepts that have been shown to be difficult. ${ }^{21}$

\section{EXPERIMENTAL DESIGN}

\section{A. Participants and environment}

The study took place in a traditional large-scale introductory algebra-based physics course at a large research university. The course was the second semester of a two-semester sequence covering electricity, magnetism, optics, and modern physics. Students, typically in their second or third year of study, received five credit hours for participating in three lectures and one integrated two-hour laboratory and recitation section per week. Weekly homework assignments were the traditional end-of-chapter style questions, presented and graded by a computer system Computer Assisted Personalized Approach (CAPA). ${ }^{22}$ Two instructors and seven teaching assistants (TAs) were assigned to the 363 students enrolled in this 15-week course.

The laboratories occurred every other week, alternating with the recitation sections. Six laboratory sessions were offered over the course of the term. The authors had recently revised the laboratories in order to emphasize an inquirybased approach. The labs stressed discovery rather than verification" $3,23,24$ and included some elements of "Scientific Community Labs." 25 This study was conducted in the second laboratory of the course, dc circuits. Students engaged in a series of exercises including examining resistors in series and parallel, building a simple circuit and then predicting, observing and reconciling its behavior as various elements (resistors or light bulbs) were added or rearranged, and finally developing methods to measure resistance in multiple ways in these circuits. The goals of the laboratory were for students to develop an understanding of simple circuits (the concepts of voltage, current, and series, parallel, and equivalent resistance), to develop the skills associated with connecting light bulbs, resistors, and wires in various combinations, and to collect data and make arguments about these circuits' behaviors. Each laboratory began with students turning in prelaboratory work and asking TAs questions about the material. 
A second, calculus-based introductory physics course served as a secondary control group for the study. The calculus-based course is composed mostly of students who plan on becoming physics or engineering majors. The course meets for four credit hours per week, three in lecture and one in recitation. Notably, there is no laboratory portion of this course. The laboratory is a separate course and was taken concurrently by approximately half of the students in this study, though none of these students had yet been formally exposed to an electric circuits laboratory in college. Data from this course were collected in four separate sections $(N=107)$ of the traditional TA-led one-hour recitation section. At the time of this study, students had attended three lectures on basic dc circuits (one of which included a demonstration of the circuit used in the challenge described below) and had a homework set covering de circuits. This class, which engages in the same challenge without the associated laboratory preparation, serves to demonstrate that the laboratory itself has an effect overall.

\section{B. Procedure}

Each of the 15 sections of the algebra-based introductory physics course completed the dc circuits lab. The sections were split into two experimental conditions: CCK (four sections; $N=99$ students), which performed the laboratory with the computer simulation, and traditional conditions, TRAD (six sections; $N=132$ students), which performed the laboratory with real equipment. (The remaining five sections participated in the laboratory in slightly varied conditions, but are not included in this study.) The test and control conditions were assigned in order to isolate effects of the TA and to include similar cross sections of students in the course. Most of the teaching assistants were assigned one section of each type (CCK and TRAD). The grade distribution of students in each condition of the study was indistinguishable from that of the course overall.

The dc circuits laboratory was nearly identical for the two groups. The written introduction to the physical equipment was the same for both groups, each receiving instructions on reading resistors, etc. Additional instructions on operating the simulation were provided to the CCK group. Each student was assigned a prelaboratory activity, which varied by group (CCK or TRAD). Three of the four questions on the prelaboratory were identical. The fourth question, which asked students to light a light bulb with a battery and a single wire, varied. The traditional group was asked to draw a circuit that would light the bulb; the CCK students were asked to build the circuit using the simulation. All students turned in their assignments at the beginning of their laboratory sections and received credit for finishing the prelaboratory; however, the prelaboratory assignments were neither graded nor returned. The actual laboratory activities (challenges and wording) were the same except the traditional groups were instructed to manipulate real equipment, and the CCK groups to manipulate simulated equipment. Students worked in groups of two to five.

The last 30 minutes of each two-hour laboratory section were set aside for students to complete a challenge work-
Questions 1, 2, and 3 refer to the circuit below in which four identical light bulbs are connected to a battery. The switch $S$ is initially closed (conducting) as shown.

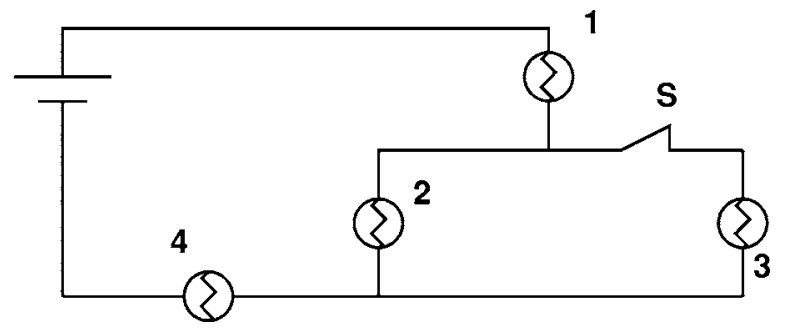

FIG. 2. Schematic diagram on final examination relating to questions about circuits.

sheet consisting of three questions. The challenges were the same for all students in this study. The main challenge was to build a circuit (the same as shown in Fig. 2) using real equipment, and to describe and explain what happened (and why) when the circuit was broken at the switch. Notably, the CCK group had no formal exposure to this equipment before this challenge. As an added control measure to confirm that the labs did affect student mastery of circuits, this same challenge sheet was given to students in the calculus-based introductory sequence (electricity and magnetism) who had no laboratory.

For the students enrolled in the algebra-based physics course, three questions addressing dc circuits were placed on the final examination to probe their mastery of current, voltage, and equivalent resistance. The final examination was issued 12 weeks after the laboratory. The exam questions referred to a circuit identical to the one built in their challenge, in schematic form, shown in Fig. 2. Students were asked to (q1) rank the currents through each of the bulbs, (q2) rank the voltage drops across the bulbs in the circuit, and (q3) predict whether the current through the first bulb increased, decreased, or remained the same when the switch was opened. ${ }^{26}$

\section{Data collection}

The following data were collected and analyzed during the course of this study.

(1) Observational notes of the sessions (both by TAs and by researchers in this study).

(2) Timing data: how long it took students to build the assigned challenge circuit as a group and write up the results individually.

(3) Laboratory challenge writeups (written up and turned in by each student).

(4) Performance on three questions on dc circuits on the final examination.

\section{RESULTS}

\section{A. Circuit challenge}

At the end of each laboratory section, all students completed the challenge worksheet in which they were asked to build a circuit using real equipment with their groups (shown 


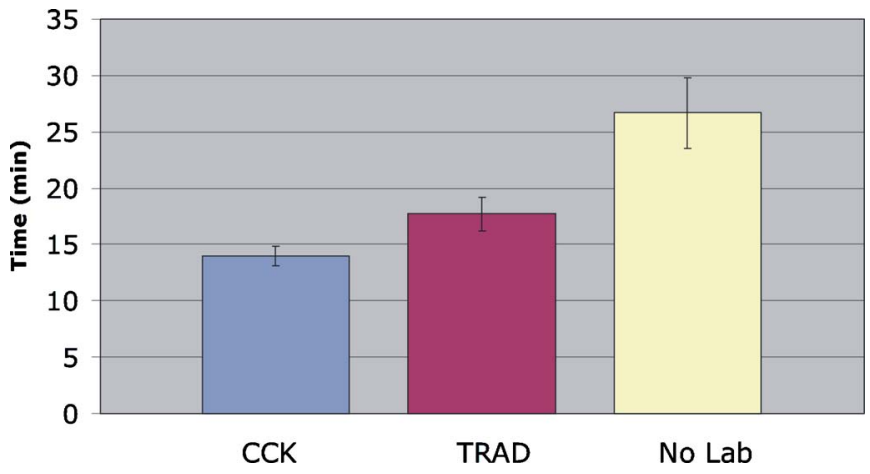

FIG. 3. (Color) Timing data showing how long it took students to build a real circuit and write up what happened. Students had previously conducted a laboratory using simulated (CCK) or real equipment (TRAD), or had not conducted a laboratory (No Lab). CCK and TRAD students are in an algebra-based course, the No Lab students are enrolled in a calculus-based course. Error bars indicate standard error of the mean for each condition.

in Fig. 2), showed it to the TA, and then broke the circuit at the point designated by the switch. Students followed by answering a short essay question on the circuit and turned in their answers individually.

\section{Timing}

TAs were given observation sheets asking them to report the amount of time students needed to complete the circuit challenge. TAs reported the average time for their entire section and the times for the fastest and slowest groups. The timing data - how long it took groups of students to build the circuit, break the circuit and write up the challenge-are plotted in Fig. 3. The averages for the experimental condition (CCK), the group using traditional equipment (TRAD), and the calculus-based course sections with no laboratory (No $\mathrm{Lab})$ are plotted. The average time to complete the circuit challenge for all CCK sections was $14.0 \mathrm{~min}$, for the traditional sections was $17.7 \mathrm{~min}$, and for the calculus-based control group was $26.7 \mathrm{~min}$. The error bars on the graph depict the standard error of the mean for each of the three experimental conditions. If we consider only the averaged data for each laboratory section participating in the study, we find the CCK group statistically faster than the TRAD group at the $p<0.1$ level (two-tailed $t$-test, across ten sections). However, if we include data on the fastest and slowest groups within each section, and assume that these times serve as an upper bound estimate for the standard deviation within each section, we find an increase in the statistical significance of the difference between average times for CCK and TRAD conditions to a $p<0.01$ level (two-tailed $t$ test, using pooled variance across 65 groups). Considered in this manner, the statistical differences in mean times between the No Lab condition and either the CCK or TRAD condition are even more significant.

\section{Evaluation of student writeups}

Each student in the circuit challenge completed a writeup answering the following question. "Describe what happens

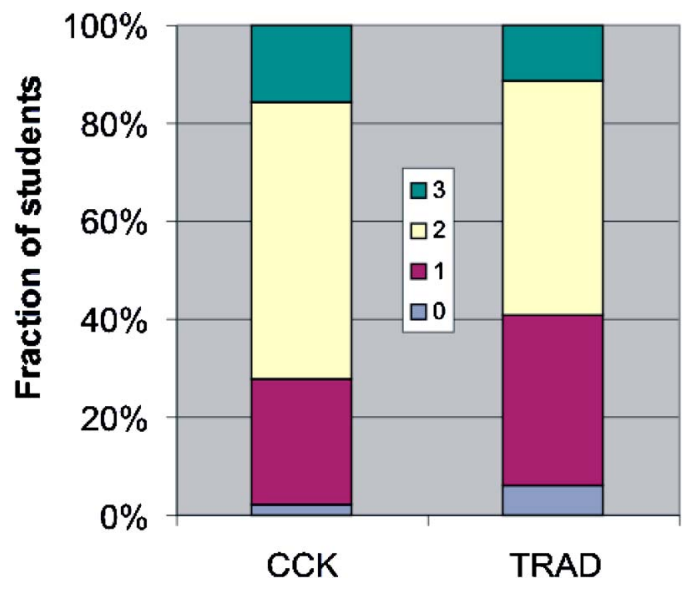

FIG. 4. (Color) Evaluation of student explanations of a circuit challenge using real equipment for students who had previously conducted a laboratory using simulations (CCK) or using real equipment (TRAD).

and WHY the bulbs change brightness as they do. You may use words and formulas. Present your reasoning in everyday language so that a friend who has never taken physics would understand your reasoning for why you ranked the bulbs as you did. (You can use words like voltage difference, current, energy, etc.)"

The answers were evaluated by the authors as to their overall correctness using a standardized rubric with a scale from 0 to 3. Zero represented no demonstrated knowledge of the domain, while 3 represented correct and complete reasoning. The research team came to consensus on the grading metric, grading not only for overall correctness, but also for use of particular concepts (current, voltage, power, series or parallel resistance) and mathematics. The authors verified agreement on select questions to ensure significant inter-rater reliability. The fractions of students scoring $0,1,2$, and 3 are reported in Fig. 4 for both the experimental group (CCK) and the traditional group (TRAD).

The average score for the experimental group (CCK) was 1.86 and for the real-equipment group (TRAD) was 1.64-a statistically significant shift $(p<0.03$, two-tailed $z$ test $)$. While these data have been analyzed by student use of particular concepts and use of mathematics (not reported here), the most significant difference between the CCK and TRAD groups was in their averaged total scores, suggesting that the CCK students were better able to integrate these concepts, a necessary ability for describing why bulbs change brightness as they do.

\section{Final examination}

Referring to a schematic drawing of a circuit with series and parallel segments (Fig. 2), students were asked to (q1) rank the currents through each of the bulbs, (q2) rank the voltage drops across the bulbs in the same circuit, and (q3) predict whether the current through the first bulb increased, decreased, or remained the same when the switch was opened. Figure 5 plots student performance for the experimental (CCK) and the traditional (TRAD) group for each of these three questions (q1, q2, q3), and their performance on 


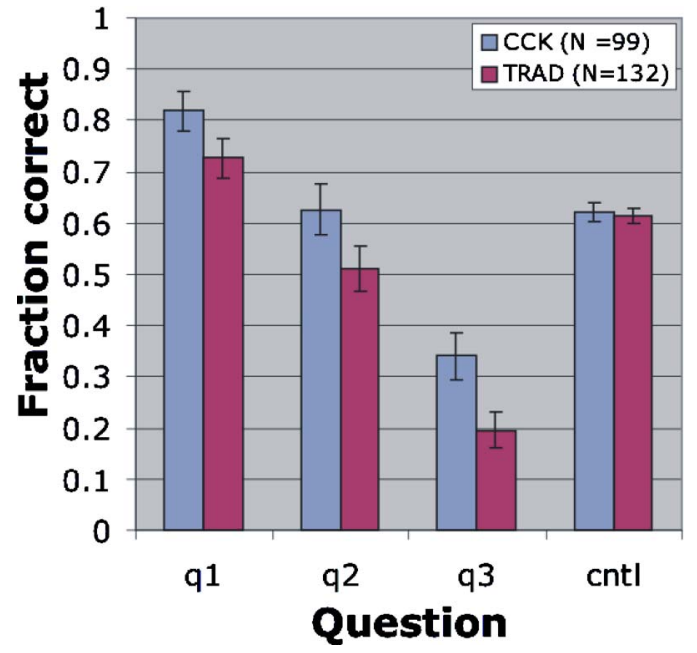

FIG. 5. (Color) Student performance on three final examination dc circuits questions (q1, q2, q3) and the remaining 26 questions on the exam (cntl), for each of two conditions: students who had conducted the circuits lab with virtual $(\mathrm{CCK})$ or real equipment (TRAD).

the remaining 26 questions on the final, which covered other material in the course (labeled "cntl"). The average on the final exam questions not relating to the circuits questions was $0.621(N=99 ; \sigma=0.18)$ for $\mathrm{CCK}$ and $0.612(N=132 ; \sigma$ $=0.17)$ for TRAD. The mean performance on the three circuits questions was $0.593(\sigma=0.27)$ for $\mathrm{CCK}$ and $0.476(\sigma$ $=0.27$ ) for TRAD groups. ${ }^{27}$ The two groups are statistically identical on the noncircuits questions and significantly different on the circuit questions, $p<0.002$ (by Fisher test or onetailed binomial distribution ${ }^{28}$ ).

\section{B. Themes from observations}

During the laboratories both graduate teaching assistants and researchers on this project observed students' interactions with each other, the TAs, and the equipment. Notes written after the observations provide some insights into the conditions and processes that explain how and why these laboratories differed with the use of computer simulations. While not an exhaustive case study of these laboratories, drawing from the field notes and observations we find particular themes arise that help delineate the differences and similarities of the two laboratory conditions. Below we include excerpts from researcher field notes collected during the study from $2 / 9 / 04$ to $2 / 15 / 04$.

\section{Students messing about}

Researchers in education and in physics have described the benefits of "messing about." 29,30 This idea of scientific play is the methodical investigation of the constraints and opportunities of a system. This play can lead to the organization of students' knowledge and its alignment with scientific models. Depending upon how these tools (in our case, simulation or real equipment) are used, messing about may or may not be productive. With the simulation, the observers note that the messing about was more generally restricted to building circuits (an activity that is considered generally on task, or supportive of the goals of the laboratory). A researcher notes:

"Three groups are already playing with CCK....still messing with resistor combinations. The TA told them to use simpler circuit combinations.... group has resistors in series and an ammeter in series"

or

"One group started but not following directions. They were playing making circuits and watching electrons flow."

Similarly, students using the real equipment were observed to mess about making circuits:

"Another group had the power supply attached to the resistor and were trying to measure the resistor with the DMM. They were getting 0 on the DMM. I asked why it was plugged into the power supply."

Less productive pursuits with real equipment, however, such as making bracelets out of the wires, were also observed.

\section{Design of the equipment}

In both the cases of the simulation and the real equipment, the tools are designed with specific constraints. These constraints can either be productive or not. For the real equipment, both the wire color and observation of a dim bulb in a bright room cause problems for students:

"Group 1 is talking about 'black and red' they have asked 2 times for more red and black wires. They have a mess of wires plugged into 1 bulb (no bulbs lit)."

or

"The circuit [for this group] is right, but they don't think the 2 parallel bulbs are glowing. [The instructor] turns off the light and turns the voltage up and they see the bulbs are glowing after all. 'That's good' one student says."

Meanwhile, the simulation is designed to make explicit both bulb brightness and an underpinning model of electron flow.

"[This] group notices electrons flowing opposite to the direction of 'current' flow. [The students] ask the TA about this and eventually realize that these blue dots must be showing electron flow (negative charge)."

No instances were reported in which students failed to recognize a bulb as lit in the virtual case, whereas this difficulty frequently arose with the real equipment. There were instances where TAs reported difficulty with the simulation, though these instances were rare. ${ }^{31}$

\section{Use of instructor time}

How the TAs spent time in class varied. In the case of the simulation, the students had been asked to conduct the prelaboratory online, and were familiar with the simulation upon arrival at the laboratory. Meanwhile, the TA in the laboratory using real equipment was frequently fetching bulbs, or 
troubleshooting unexpected circumstances (e.g., dim bulbs that had been misinterpreted as unlit). One observer captured these differences in describing the CCK laboratory:

"compared to [my experiences in this same] electronics lab (2 years ago), this lab was calm and composed. I remember the lab being fairly chaotic, having to run about a lot with students asking questions constantly. ... today the $[\mathrm{TA}]$ was not just sitting doing nothing, but [the TA] did not have to answer multiple students at once."

Of course, there are failure modes for the simulations as well. For instance, as described below, one section suffered from repeated computer failure, which demonstrates both the increased demands on the TA and the limitations of equipment when a simulation fails to operate as designed.

\section{Replication study}

In a subsequent study during the off-semester offering of the algebra-based introductory physics course, the same experiment was repeated, but with a smaller number of students $(N=80)$. The same laboratories were run using either real or simulated equipment, and students completed the same challenge activity. The same trends were observed in student performance: A higher fraction of students using the CCK simulation correctly answered the conceptual questions about current and voltage than their counterparts who used real equipment. Furthermore, all sets of students assembled the challenge circuit and answered questions in a similar amount of time. Because of the smaller sample size, the statistical and systematic uncertainties are greater. Additionally, we found that the variation in time between different sections was greater than the variation between treatment $(\mathrm{CCK})$ and control (TRAD) groups. One reason for this was that in this instance of the study, one of the two sections using the simulation encountered significant computer difficulties. Specifically, the bulk of the computers would freeze up in the midst of the simulation. (This glitch was inadvertently caused by minor upgrades to the simulation after the first study, which resulted in these unexpected problems. The simulation is now fixed.) By alerting students and teaching assistants to the conditions that would cause their computers to seize, the second laboratory section using the simulations did not encounter this problem and students in this section reported the fastest times at assembling the challenge circuits and higher scores than their counterparts (both those using real equipment and those who had computer difficulties) on the conceptual questions.

\section{Discussion}

The present study suggests that it is possible, and in the right conditions preferable, to substitute virtual equipment for real laboratory equipment. Of course, there are many constraints on when and how this might occur, including but not limited to considerations of the context into which these simulations are embedded (both pedagogically and logistically). ${ }^{32} \mathrm{We}$ are not claiming that all circuits labs ought to be replaced, but rather the conventional wisdom that students learn more via hands-on experience is not borne out by measures of student performance on assessment of conceptual understanding, nor by their ability to construct circuits. ${ }^{33}$ In an inquiry-based laboratory, students using the simulations learned more content than did students using real equipment.

Notably, the results on the final examination demonstrate that the students who used the simulation had a better mastery of current, voltage, and resistance in basic dc circuits. Furthermore, this mastery was demonstrated roughly more than two months after the laboratory was conducted on the final (after many other forms of exposure to the same material, including lectures, homework, exams, and studying).

No less significant, student facility in constructing real circuits is supported by the simulations. The data suggest that students who have worked with simulations are more capable at constructing and writing about circuits than their counterparts who have been working with the physical apparatus all along. In addition to more correctly and thoroughly writing about the circuits, the students take less time on average than their counterparts at building and describing these circuits. The added control group (the calculus-based section) corroborates that the lab itself supports student capabilities with circuits, whether using real or virtual equipment; however, in this case, we find that the virtual experience results in the greater student facility with circuits.

It is worth noting that computers are subject to the same vagaries of breaking down that plague real equipment. They can operate in unintended ways, and frustrate users as much as any mechanical or electrical device can. Furthermore, other researchers ${ }^{34}$ note concerns that new tools may provide new educational difficulties. In a study of how students use multimedia tools in an algebra-based college class, Yeo et $a l .{ }^{34}$ "question the efficacy of instruction and interactive multimedia programming ... which allows student to move, without question, from one difficult concept to another in as short a time as 60 s." Later they note that it is important that "appropriately timed 'reflective' points could be incorporated in the program enabling students to self-assess their understanding of concepts. Students should not be able to simply exit a screen with alternative concepts left intact (or reinforced)." These concerns have more to do with how new media are used in the classroom, that is, with the pedagogy, rather than with the tools themselves. In the end, we argue that these new media may provide better mechanisms for ensuring appropriate pedagogy than other hands-on and pencil-and-paper activities.

Others have suggested a list of important characteristics of computer-based activities and simulations-from actively engaging the students to providing tools to reduce unnecessary drudgery. ${ }^{3,8}$ To this list we add two more items which begin to explain the present findings. As discussed below, computers can

(1) increase student access to productive concepts and representations, and

(2) constrain the students in productive ways.

A variety of visual cues in the computer simulations make concepts visible that are otherwise invisible to students. In the present study, most notably the simulation provides direct perceptual access to the concept of current flow. A visual representation of current is provided that allows st udents to 
study concepts that are otherwise hidden; e.g., current conservation (electrons do not get "used up" in a light bulb) and signal propagation (electrons do not drift at the signal speed). Again, while researchers continue to debate the merits of explicitly modeling microscopic behavior in these environments, ${ }^{18,19}$ we suggest that this intermediate stage, providing a visual model of current flow, is productive in this environment. Such features of accessibility are not limited to current flow; pragmatic considerations are also consideredthere is no ambiguity for students in determining whether a light bulb is on, or whether wires are connected. Clear visual cues are provided to emphasize these conditions, which otherwise can cause troubles for students using real circuits. ${ }^{35}$

While it may seem counterintuitive, the limited nature of investigation afforded by a simulation can be productive. That is, because the system under investigation is constrained in particular ways, students are able to make progress they cannot in an unconstrained environment. For example, students are not given the choice of wire color and hence do not attribute meaning to insulator color in the simulation. In the case of real equipment, wire color can serve as a distraction. Simulations provide the instructor considerably more freedom in designing and applying constraints to ensure that students' messing about leads to productive learning. Just as physicists seek to isolate individual variables and constrain a system to observe the effects of that variable, so too does the simulation scaffold students' understanding, by focusing attention to relevant details. As Otero finds, students use computer simulations productively to produce conceptual models that are then effectively applied to physical ("real world") applications. ${ }^{36}$

\section{E. Conclusions}

In these studies, students who used computer simulations in lieu of real equipment performed better on conceptual questions related to simple circuits, and developed a greater facility at manipulating real components. We do not suggest that simulations necessarily promote conceptual learning nor do they ensure facility with real equipment, but rather computer simulations that are properly designed are useful tools for a variety of contexts that can promote student learning. In this particular environment, a fairly traditional dc circuits laboratory with the explicit goals of developing students' understanding of simple circuits, as well as skills at manipulating these components and reasoning about their behavior, the virtual equipment is more productive than real equipment. Computers are far from the magic bullet many look for in education. However, we demonstrate that computers are not simply useful because they are ubiquitous and expedient in environments with otherwise limited resources. Redish asks "Is the computer appropriate for teaching physics?" 37 We suspect its time may have arrived. "To simulate or not to simulate?" asks Steinberg. ${ }^{38}$ We answer yes, providing simulations are properly designed and applied in the appropriate contexts.

\section{ACKNOWLEDGMENTS}

This work was conducted with the support of the Kavli Operating Institute, the National Science Foundation, the American Physical Society's Physics Teacher Education Coalition, and the University of Colorado. The authors are grateful to Carl Wieman, the PhET team, and members of the Physics Education Research Group at Colorado (PER@C).
${ }^{1}$ D. R. Sokoloff and R. K. Thornton, Phys. Teach. 35, 340 (1997).

${ }^{2}$ R. N. Steinberg, G. E. Oberem, and L. C. McDermott, Am. J. Phys. 64, 1370 (1996).

${ }^{3}$ R. K. Thornton and D. R. Sokoloff, Am. J. Phys. 58, 858 (1990).

${ }^{4}$ A. A. diSessa, Changing Minds (MIT Press, Cambridge, MA, 2000).

${ }^{5}$ S. Papert and I. Harrel, Constructionism, edited by Idit Harel and Seymour Papert (Ablex Publishing Corporation, Norwood, NJ, 1991).

${ }^{6}$ S. Turkle, Seeing Through Computers, The American Prospect, Vol. 8, Issue 31 (1997).

${ }^{7}$ P. Laws, Phys. Today 44 (12), 24 (1991).

${ }^{8}$ E. F. Redish, J. M. Saul, and R. N. Steinberg, Am. J. Phys. 65, 45 (1997)

${ }^{9}$ R. Trumper, Science and Education 12, 645 (2003).

${ }^{10} \mathrm{http}: / /$ www.laaphysics.org

${ }^{11}$ Z. Zacharia and O. R. Anderson, Am. J. Phys. 71, 618 (2003).

${ }^{12}$ M. Linn and S. Hsi, Computers, Teachers, Peers: Science Learning Partners (Lawrence Erbaum, Mahwah, NJ, 2000).

${ }^{13}$ M. Linn, B. Eylon, and E. Davis, Internet Environments for Science Education, edited by M. Linn, E. Davis, and P. Bell (Lawrence Erbaum, Mahwah, NJ, 2004).

${ }^{14}$ L. M. Triona and D. Klahr, Cogn. Instruct. 21, 149 (2003).
${ }^{15}$ K. K. Perkins, W. K. Adams, M. Dubson, N. D. Finkelstein, R. Lemaster, S. Reid, and C. E. Wieman, Phys. Teach. (to be published).

${ }^{16} \mathrm{http}: / /$ phet.colorado.edu

${ }^{17}$ In many senses this is approaching diSessa's microworld (Ref. 4), where students are embedded in a world with internally consistent rules and open ended explorations.

${ }^{18}$ B. Eylon and U. Ganiel, Int. J. Sci. Educ. 12, 79 (1990).

${ }^{19}$ B. Thacker, U. Ganiel, and D. Boys, Am. J. Phys. 67, S25 (1999).

${ }^{20}$ C. Keller, N. Finkelstein, K. Perkins, and S. Pollock (unpublished).

${ }^{21}$ L. McDermott and P. S. Shaffer, Am. J. Phys. 60, 994 (1992).

${ }^{22} \mathrm{http}: / /$ www.lon-capa.org

${ }^{23}$ P. Laws, Workshop Physics Activity Guide, 2nd ed. (John Wiley and Sons, New York, 2004).

${ }^{24}$ L. C. McDermott, Physics by Inquiry (John Wiley and Sons, New York, 1996).

${ }^{25}$ R. Lippman, Ph.D. dissertation, University of Maryland, 2003, http://www.physics.umd.edu/rgroups/ripe/perg/dissertations

${ }^{26}$ These questions follow from those in the Electric Circuits Concept Evaluation (ECCE) and are part of Workshop Physics: http://physics.dickinson.edu (Ref. 23). 
${ }^{27}$ Notably, the poorest performance is observed on question 3 (predicting current flow through bulb 1). Researchers at the University of Washington have demonstrated that this is a particularly difficult concept to master, and students generally do not perform well (Ref. 21). However, here we make no claims about the absolute effectiveness of the curriculum, but rather the effect of using differing tools in a somewhat standard curriculum. Future studies examine the role of using these tools with other curricula (Ref. 20).

${ }^{28}$ D. Sheshkin. Handbook of Parametric and Nonparametric Statistical Procedures, 3rd ed. (CRC Press, Boca Raton, FL, 2004).

${ }^{29}$ D. Hawkins, The Informed Vision: Essays on Learning and Human Nature (Agathon Press, New York, 1974).

${ }^{30}$ D. Hammer, Am. J. Phys. 68, S52 (2000).

${ }^{31}$ For instance, one instructor reported difficulties with the virtual voltmeter, because unlike its physical counterpart, the virtual meter did not act as a multimeter and could not be set to read resistance. Until the instructor recognized this, he attempted to use the voltmeter to measure resistance.
${ }^{32}$ N. D. Finkelstein, physics/0505092, Int. J. Sci. Educ. (to be published).

${ }^{33} \mathrm{We}$ suspect there are some benefits to using real equipment, but we did not measure them here.

${ }^{34}$ S. Yeo, R. Loss, M Zadnik, A. Harrison, and D. Treagust, Am. J. Phys. 72, 1351 (2004).

${ }^{35} \mathrm{We}$ do not suggest that troubleshooting circuits is a less valuable skill. Rather, the goal of the laboratory did not focus on such skills, and so the need for students to do substantial troubleshooting prevented them from focusing on the main idea of the laboratory (to develop a model of simple circuits).

${ }^{36}$ V. Otero, in Research on Physics Education, Proceedings of the International School of Physics "Enrico Fermi," Course CLVI, edited by E. F. Redish and M. Vicentini (Italian Physical Society, Bologna, Italy, 2003).

${ }^{37}$ E. F. Redish, Comput. Phys. 7, 613 (1993).

${ }^{38}$ R. N. Steinberg, Am. J. Phys. 68, S37 (2000). 